\title{
Mixed chimerism and tolerance without whole body irradiation in a large animal model
}

\author{
Yasushi Fuchimoto, ${ }^{1}$ Christene A. Huang, ${ }^{1}$ Kazuhiko Yamada, ${ }^{1}$ Akira Shimizu, ${ }^{2}$ \\ Hiroshi Kitamura, ${ }^{2}$ R.B. Colvin, ${ }^{2}$ Vincent Ferrara, ${ }^{1}$ Michael C. Murphy, ${ }^{1}$ Megan Sykes,${ }^{1}$ \\ Mary White-Scharf, ${ }^{3}$ David M. Neville, Jr., ${ }^{4}$ and David H. Sachs ${ }^{1}$ \\ ${ }^{1}$ Transplantation Biology Research Center, and \\ ${ }^{2}$ Department of Pathology, Massachusetts General Hospital/Harvard Medical School, Boston, Massachusetts, USA \\ ${ }^{3}$ BioTransplant Inc., Boston, Massachusetts, USA \\ ${ }^{4}$ Laboratory of Molecular Biology, National Institute of Mental Health, Bethesda, Maryland, USA \\ Address correspondence to: David H. Sachs, Transplantation Biology Research Center, \\ Massachusetts General Hospital, MGH-East, Building 149-9019, 13th Street, Boston, Massachusetts 02129, USA. \\ Phone: (617) 726-4065; Fax: (617) 726-4067; E-mail: sachs@helix.mgh.harvard.edu. \\ Yasushi Fuchimoto's present address is: Tokyo Metropolitan Kiyose Children's Hospital, Tokyo, Japan. \\ Akira Shimizu's present address is: Department of Pathology, Nippon Medical School, Tokyo, Japan. \\ Yasushi Fuchimoto and Christene A. Huang contributed equally to this work.
}

Received for publication October 18, 1999, and accepted in revised form April 26, 2000.

\begin{abstract}
Mixed hematopoietic chimerism may provide a treatment for patients with nonmalignant hematologic diseases, and may tolerize patients to organ allografts without requiring chronic immunosuppression. However, the toxicity of the usual conditioning regimens has limited the clinical applicability of this approach. These regimens generally include some level of whole body irradiation (WBI), which is thought to facilitate engraftment either by making room for donor hematopoietic stem cells or by providing sufficient host immunosuppression to enable donor cells to engraft. Here, we have established mixed chimerism across both minor and major histocompatibility barriers in swine, by using high doses of peripheral blood stem cells in the absence of WBI. After mixed chimerism was established, swine leukocyte antigen-matched (SLA-matched) donor skin grafts were tolerated and maintained for a prolonged period, whereas third-party SLA-matched skin was rejected promptly. Donor-matched kidney allografts were also accepted without additional immunosuppression. Because of its low toxicity, this approach has potential for a wide range of clinical applications. Our data may indicate that niches for engrafting stem cells are filled by mass action and that WBI, which serves to empty some of these niches, can be omitted if the donor inoculum is sufficiently large and if adequate host T-cell depletion is achieved before transplant.
\end{abstract}

J. Clin. Invest. 105:1779-1789 (2000).

\section{Introduction}

There are numerous clinical situations for which bone marrow transplantation (BMT) might be the treatment of choice. However, standard preparative regimens for BMT generally include whole body irradiation (WBI) to create space (1) or sufficient immunosuppression (2) for bone marrow engraftment. Such regimens are so toxic on their own that few patients are offered this option until they are late in the course of their disease. These situations include patients with hemoglobinopathies, such as sickle cell disease and thalassemia (3-5), as well as patients with end-stage organ failure, who could be treated by allogeneic organ transplantation without the complications of long-term immunosuppression, if they could be made tolerant by $\operatorname{BMT}(6,7)$. For this reason, recent studies in this laboratory have been directed toward developing means of establishing mixed hematopoietic chimerism using nonmyeloablative and relatively nontoxic conditioning regimens.

In mice, long-term hematopoietic engraftment has been achieved in unconditioned recipients by injection of high doses of syngeneic bone marrow $(8,9)$. More recently, this approach has been extended to the achievement of hematopoietic engraftment and donorspecific tolerance across a full MHC barrier using sublethal irradiation (10) or without myelosuppressive host conditioning, by using depleting mAb's, local thymic irradiation, and very high doses of MHC-mismatched bone marrow cells (11).

These results are extremely promising for our clinical goals. However, for a variety of reasons, engraftment may be easier to achieve in mice than in large animals and humans, and many regimens that have been successful in mice have not been effective clinically (12-15). Testing in a large animal model is therefore critical to extending this nonmyelosuppressive approach to the establishment of hematopoietic chimerism for human transplantation. Stable mixed chimerism after BMT has been demonstrated in dogs, using dog leukocyte antigen-identical (DLA-identical) littermates $(2,16)$. Recently we have also been successful in establishing stable mixed chimerism across 
swine leukocyte antigen-matched (SLA-matched) barriers in sublethally irradiated miniature swine (17). To date, mixed chimerism has not been achieved across full MHC barriers in large animal models without significant myelosuppression.

In the present study, we have extended our nonmyeloablative protocol, making it nonmyelosuppressive, by eliminating WBI from the conditioning regimen used to allow hematopoietic cell transplantation. Transplants were performed across full major histocompatibility antigen mismatches, as well as across minor histocompatibility antigen mismatches, using high doses of peripheral blood stem cell (PBSC) as a source of hematopoietic cells. Tolerance was confirmed in these animals by skin grafting or transplantation of donormatched kidney allografts.

\section{Methods}

Animals. Transplant donors and recipients were selected from our herd of MGH partially inbred, MHC-defined miniature swine. PBSC donors were 4-8 months old and weighed $30-50 \mathrm{~kg}$. Recipients were 8-12 weeks old and weighed $5-15 \mathrm{~kg}$. The immunogenetic characteristics of this herd and intraMHC recombinant haplotypes have been described previously $(18,19)$.

PBSC collection and infusion. PBSC collection has been described previously $(20,21)$. A stem cell-mobilizing regimen consisting of recombinant porcine stem cell factor ( $p S C F ; 100 \mu \mathrm{g} / \mathrm{kg}$ ) in combination with recombinant porcine-IL-3 ( $p$ IL-3; $100 \mu \mathrm{g} / \mathrm{kg}$ ) (both from Biotransplant, Boston, Massachusetts, USA), with or without recombinant human G-CSF (rbu G-CSF; Amgen Inc., Thousand Oaks, California, USA), was administered subcutaneously. Collection of PBSCs was achieved by leukapheresis (COBE BCT Inc., Lakewood, Colorado, USA) beginning on day 5 of cytokine therapy and continuing daily for 3 or 6 days. PBSC, either fresh or frozen and quickly thawed, were adjusted to a concentration of $2.0 \times 10^{8} / \mathrm{mL}$, and the appropriate volume was infused through the intravenous line over a 15 - to 20 -minute period. PBSCs were administered on day 0 .

Thymic irradiation. Thymic irradiation (TI) was administered on day -2 . Irradiation was administered to a single field encompassing the thymus, defined by an $x$-axis including the width of the jaw and a $y$-axis including the distance from the top of the sternum to the temporomandibular joint. The average radiation field size was $10 \times 4 \mathrm{~cm}$ with a source separation distance of $78 \mathrm{~cm}$. The defined field was irradiated with $700 \mathrm{cGy}$ or 1,000 cGy from a cobalt irradiator source at a rate of $175 \mathrm{cGy} / \mathrm{min}$ and to a tissue depth of $2 \mathrm{~cm}$.

Peripheral blood T-cell depletion. T-cell depletion was achieved using the reagent $\mathrm{PCD} 3-\mathrm{CRM} 9$, a new mutant Diphtheria toxin-anti-swineCD3 conjugate (22). On day $-2,0.05 \mathrm{mg} / \mathrm{kg}$ pCD3-CRM9 was administered intravenously to recipient animals.
CyA treatment. Recipients received CyA through a gastric tube (Neoral; Novartis Pharmaceuticals, East Hanover, New Jersey, USA) at $15-30 \mathrm{mg} / \mathrm{kg} / \mathrm{d}$ in divided doses from day -1 to day 30 to maintain a blood level of $300-800 \mathrm{ng} / \mathrm{mL}$.

Antibodies and flow cytometry. The following swine-specific antibodies were used to monitor depletion and recovery of cell populations in the pig by flow cytometry after PCD3-CRM9 administration and PBSC infusion: anti-CD1 mouse mAb 76-7-4 (mouse IgG2aK) (23); antiCD3 mouse mAb 898H2-6-15 (mouse IgG2aK) (24); anti-CD3a mAb BB23-8E6 IgG1, and anti-CD5 mAb BB6-9G12 IgG1 provided by M. Pescovitz (Indiana University, Indianapolis, Indiana, USA; ref. 25). Flow cytometry was performed using a Becton Dickinson FACScan (San Jose, California, USA). Unseparated heparinized peripheral blood $(\mathrm{PB})$ was distributed into staining tubes (Falcon 2054) at $100 \mu \mathrm{L} /$ tube and washed twice using 2 $\mathrm{mL}$ flow cytometry buffer (HBSS containing $\mathrm{Ca}^{2+}$ and $\mathrm{Mg}^{2+} / 0.1 \% \mathrm{BSA} / 0.1 \% \mathrm{NaN}_{3}$ ). Cells were stained with optimal concentrations of the primary mouse anti-pig $\mathrm{mAb}$ (either unconjugated or FITC conjugated) for 30 minutes at room temperature. For indirect staining, goat anti-mouse Ig (H\&L) FITC (GAMF) (Sigma Immunochemicals, St. Louis, Missouri, USA) was added for an additional 30 minutes at room temperature. For detection of immunotoxin-coated cells, second step reagent alone was added. To determine the percent of donor cells among peripheral blood cell populations, $\mathrm{PB}$ was incubated with the pig monocyte/granulocyte-specific FITCconjugated mAb SWC3a 74-22-15 (Balb/c, IgG1K) (23) together with either the donor-specific biotin-conjugated mAb 1038H-10-9 (B10.PD1, IgMK) specific for pig allelic antigen (PAA) (26) or an IgM isotype-matched negative control $\mathrm{mAb}$, followed by phycoerythrin streptavidin (PESA; PharMingen, San Diego, California, USA). Red blood cells were lysed, and the white cells were fixed using FACS lysing solution (Becton Dickinson) before acquisition. Data were analyzed using Winlist list mode analysis software (Verity Software House, Topsham, Maine, USA). For flow cytometry of thymus cell suspensions, cells were distributed into tubes at $1 \times 10^{6}$ cells per tube. Staining was identical to that already described here except that no FACS lysing solution was added. Propidium iodide was added immediately before acquisition in order to exclude dead cells.

Histology. Thymic tissues were obtained by sequential biopsies. Formalin-fixed tissue sections were stained with hematoxylin and eosin (H\&E) and examined microscopically. For immunohistochemical study of thymus in animals receiving single haplotype-mismatched PBSC, frozen tissues were used, and sections were stained with the mAb16-7-D4 (27), specific for $\mathrm{SLA}^{c}$ class I (donor type), using indirect immunoperoxidase technique. To identify the lineage of SLA ${ }^{c}$ class I-positive cells (donor cells), frozen tissues were stained using sequential immunoperoxidase and immunoalkaline phosphatase techniques to detect keratin (DAKO A/S, Glastrup, Denmark) or ISCR 3, 


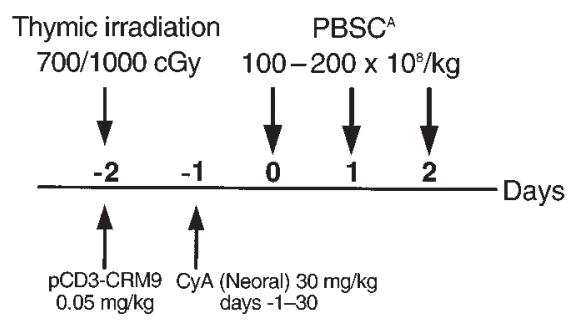

Figure 1

Diagram of preparative regimen used for PBSCT. ${ }^{A} A n$ additional 3 days of leukapheresis were necessary to yield $200 \times 10^{8} / \mathrm{kg}$ for animal no. 13101.

specific for SLA class II DR. Control stains were performed by substitution of the primary antibody with an irrelevant murine $\mathrm{mAb}$.

Skin grafts. Skin grafts were performed by techniques published previously (28). Briefly, split-thickness skin was harvested from the donor and placed on a deep split-thickness bed on the recipient's dorsal thorax. Grafts were examined daily until rejection occurred. Rejection was determined macroscopically and defined as diffuse cyanosis and induration of the graft.

Kidney transplantation. The surgical procedures used for kidney transplants have been described in detail previously $(29,30)$. A semipermanent indwelling Hickman silastic central venous catheter was placed surgically into the external jugular vein. The catheter facilitated CyA administration and frequent blood sampling for monitoring of renal function, and in vitro assays. Rejection was monitored by plasma creatinine tests and histological examination of biopsy tissue.

Preparation of PBMCs. For separation of PBMCs, freshly heparinized whole blood was diluted 1:2 with HBSS (GIBCO BRL, Gaithersburg, Maryland, USA), and mononuclear cells were obtained by gradient centrifugation using lymphocyte separation medium (Organon Teknika, Durham, North Carolina, USA). The mononuclear cells were washed once with HBSS, and contaminating red cells were lysed with ammonium chloride potassium buffer (B\&B Research Laboratory, Fiskeville, Rhode Island, USA). Cells were then washed with HBSS and resuspended in tissue culture medium. All cell suspensions were kept at $4{ }^{\circ} \mathrm{C}$ until used in cellular assays.
Cell-mediated lympholysis. Media for cell-mediated lympholysis (CML) cultures consisted of RPMI 1640 (GIBCO BRL) supplemented with 6\% FCS (Sigma Chemical Co., St. Louis, Missouri, USA), $100 \mathrm{U} / \mathrm{mL}$ penicillin, $135 \mu \mathrm{g} / \mathrm{mL}$ streptomycin (GIBCO BRL), 50 $\mu \mathrm{g} / \mathrm{mL}$ gentamicin (GIBCO BRL), $10 \mathrm{mM}$ HEPES (Fisher Scientific, Pittsburgh, Pennsylvania, USA), 2 $\mathrm{mM}$ L-glutamine (GIBCO BRL), $1 \mathrm{mM}$ sodium pyruvate (BioWhittaker Inc., Walkersville, Maryland, USA), and $5 \times 10^{-5} \mathrm{M} \beta-2$ mercaptoethanol (Sigma Chemical Co.). Media used for the effector phase of CML assays consisted of Basal Medium Eagle (GIBCO BRL) supplemented with 6\% serum replacement medium (Sigma Chemical Co.). CML assays were performed as described previously $(29,30)$. Briefly, lymphocyte cultures containing $4 \times 10^{6}$ responder and $4 \times 10^{6}$ irradiated $(25 \mathrm{~Gy})$ stimulator PBMCs in $2 \mathrm{~mL}$ of medium were incubated for 6 days at $37^{\circ} \mathrm{C}$ in $7 \% \mathrm{CO}_{2}$ and $100 \%$ humidity. Bulk cultures were harvested, and effector cells were tested on ${ }^{51} \mathrm{Cr}$-labeled PHA blasts. The tests were run at serially diluted effector/target ratios (100:1, 50:1, 25:1, 12:1). After 5.5 hours of effector cell incubation with the $5 \times 10^{3}$ targets, the supernatants were harvested and ${ }^{51} \mathrm{Cr}$ release was determined on a gamma counter. Baseline levels were measured as the rate of spontaneous release of ${ }^{51} \mathrm{Cr}$ from $5 \times 10^{3}$ targets. The results were expressed as percentage specific lysis: \% specific lysis = experimental release $(\mathrm{cpm})-$ spontaneous release $(\mathrm{cpm}) /$ maximum release $(\mathrm{cpm})$ - spontaneous release $(\mathrm{cpm})] \times 100$.

\section{Results}

Conditioning regimen. Animals were conditioned with a nontoxic protocol consisting of TI and in vivo T-cell depletion before donor cell infusion. The dose of TI and donor PBSCs were increased during the course of these experiments to achieve consistent engraftment across full mismatch barriers. Figure 1 is a schematic diagram of the preparative regimen for hematopoietic cell transplantation that was used in all subsequently transplanted animals. A summary of animals receiving PBSC transplantation (PBSCT) under this protocol is shown in Table 1.

PBSC mobilization and collection. We have recently demonstrated that a combination of porcine $p \mathrm{SCF}$, pIL-3, and human G-CSF could be used to mobilized

Table 1

Summary of animals treated with nonmyelosuppressive regimen plus PBSCT

\begin{tabular}{|c|c|c|c|c|c|c|}
\hline Animal no. & SLA & $\begin{array}{l}\text { Thymic irradiation } \\
\text { (cGy) }\end{array}$ & $\begin{array}{l}\text { PBSC dose } \\
\left(\times 10^{8} / \mathrm{kg}\right)\end{array}$ & $\begin{array}{c}\text { Engraftment } \\
\text { Thymic chimerism }\end{array}$ & GvHD & $\begin{array}{l}\text { Clinical } \\
\text { outcome }\end{array}$ \\
\hline 12757 & $\mathrm{ac} \rightarrow \mathrm{ac}$ & 700 & $110^{\mathrm{A}}$ & Yes & No & Healthy \\
\hline 12963 & $c c \rightarrow c c$ & 700 & $100^{\mathrm{A}}$ & Yes & No & Healthy \\
\hline 12885 & $\mathrm{ac} \rightarrow \mathrm{ad}$ & 700 & 100 & No & No & Healthy \\
\hline 13476 & $\mathrm{~cd} \rightarrow \mathrm{ad}$ & 700 & 150 & Yes & No & Healthy \\
\hline 13100 & $\mathrm{ac} \rightarrow \mathrm{ad}$ & 1000 & 100 & Yes & Yes & Sacrificed day 73 \\
\hline 13101 & $\mathrm{ac} \rightarrow \mathrm{ad}$ & 1000 & 200 & Yes & Yes & Healthy \\
\hline
\end{tabular}

Miniature swine received thymic irradiation and $0.05 \mathrm{mg} / \mathrm{kg} \mathrm{pCD3-CRM9}$ followed by PBSC transplantation with a 30-day course of CyA. ${ }^{\mathrm{A} D o n o r}$ PBSCs were frozen and thawed prior to infusion. All other transplants were done using fresh donor cells. 


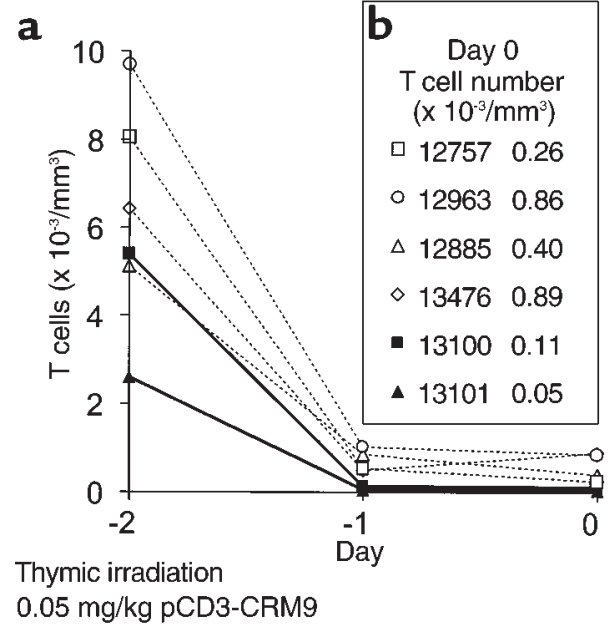

Figure 2

Peripheral blood T-cell depletion. (a) Absolute number of T cells present in the peripheral blood pretreatment (day -2), and post $\mathrm{TI}$ (700 or 1,000 cGy) and pCD3-CRM9 administration (days -1 and 0 ). (b) Absolute number of T cells remaining in PB on day 0 for each animal.

stem cell progenitors into the peripheral blood of miniature swine and that stem cell progenitors mobilized under these conditions were capable of reconstituting a lethally irradiated animal (20). After cytokine treatments, white blood cell counts in one representative donor animal rapidly rose to a peak of approximately $80 \times 10^{3} / \mathrm{mm}^{3}$. PBSCs were collected by leukapheresis on days $5-7$, and $18 \times 10^{10}$ mononuclear cells were collected. This mobilization procedure thus provides more than 10 times the number of cells achieved by bone marrow harvest from vertebral bodies and long bones (31) and more than 50 times the number of cells achievable by harvests from living donors (31).
Peripheral blood T-cell depletion in conditioned recipients. The establishment of mixed chimerism depends on effective in vivo T-cell depletion of the recipient (22). Figure 2 shows the course of peripheral blood T-cell depletion from pretreatment to the time of donor cell infusion. More than $90 \%$ peripheral blood T-cell depletion was achieved by day 0 in animals that received 700 cGy TI and anti-CD3 immunotoxin, and more than $98 \%$ T-cell depletion was achieved by day 0 in animals that received 1,000 cGy TI and immunotoxin. In addition, the few remaining peripheral $\mathrm{T}$ cells were almost entirely coated with antibody by day 0 . It should be noted that the vascular leak syndrome associated with immunotoxin treatment $(32,33)$ has never been observed in this protocol.

Lack of significant myelosuppression and organ toxicity in conditioned recipients. Potential toxicities associated with this approach were monitored by several parameters, including daily complete blood count and weekly creatinine and liver function tests, including serum glutamic oxaloacetic transaminase and serum glutamic pyruvic transaminase. No toxicity was observed due to the treatment regimen, and both renal and liver function tests were consistently normal except during the brief documented episodes of graft versus host disease (GvHD), which occurred in animals nos. 13100 and 13101 . Figure 3 shows the white blood cell (WBC) and platelet counts in the six animals treated with T-cell depletion and either 700 cGy or 1,000 cGy TI. The nadir WBC count generally occurred on day 0 and was $2,000-7,000 / \mu \mathrm{L}$. A rapid increase in WBC count and stabilization at more than $5,000 / \mu \mathrm{L}$ occurred within $2-4$ days (Figure $3 \mathrm{a}$ ). Thus, clinically significant leucopenia did not follow conditioning for PBSC transplantation (PBSCT). The platelet level was generally maintained at more than


Figure 3

Clinical course of animals receiving (700 or 1,000 cGy TI + DTCD3) on WBC (a) and platelets (PLT) (b). 
Table 2

Survival of skin grafts of SLA-identical mixed chimeric pigs

\begin{tabular}{lcc}
\hline Animal no. & Donor skin & 3P MHC-matched skin \\
12757 & $>300$ & 9 \\
12963 & 45 & $9,9^{\mathrm{A}}$ \\
\hline
\end{tabular}

AAnimal no. 12963 received two different MHC-matched third-party (3P) skin grafts.

$100,000 / \mu \mathrm{L}$, and platelet infusions were not required (Figure $3 \mathrm{~b}$ ). The general condition of the animals receiving this treatment regimen was very stable, and infection was never observed.

Analysis of engraftment and tolerance in animals receiving high-dose SLA-identical PBSC infusion

Multilineage peripheral blood chimerism. Chimerism was measured by flow cytometry using our PAA mAb, which recognizes a pig allelic antigen present on all swine leukocytes (26). Donor and recipient animals were selected that were $\mathrm{PAA}+$ and $\mathrm{PAA}-$, respectively, to facilitate chimerism detection. Animals nos. 12757 and 12963 were treated with 700 cGy TI, pCD3-CRM9 $0.05 \mathrm{mg} / \mathrm{kg}, 30$ days of CyA administration, and $100 \times 10^{8} / \mathrm{kg}$ SLA-identical PBSC infusion. In both animals, lymphocyte chimerism was achieved and rose rapidly after day 20, peaking at approximately $40 \%$ (Figure $4 \mathrm{a}$ ). One animal showed a transient decrease in lymphocyte chimerism after cessation of CyA treatment. In both animals, chimerism gradually decreased at days 40-60, reaching a stable level of $15-20 \%$, which was maintained for more than 300 days. Chimerism in the myeloid (monocyte and granulocyte) lineages was present at the $5-10 \%$ level and was also maintained for more than 300 days.

Thymic chimerism. Thymic biopsies taken in the first 2 weeks revealed an atrophic thymus in both animals after TI (data not shown). By 20 days after transplant, the thymus began to grow, as assessed by visualization at biopsy. Thymic chimerism increased rapidly at this time, peaking on day 45. Thereafter, thymic chimerism decreased, stabilizing at $5-10 \%$ by day 60 and remaining at that level for more than 300 days in both animals (Figure 4b).

Skin grafts. Skin grafts were performed on day 60 to test for the establishment of donor-specific tolerance. One animal accepted the donor skin graft indefinitely (> 250 days), and the other animal showed prolonged donor skin graft survival, but rejected on day 45. Both animals rejected $\mathrm{MHC}$ matched third-party skin grafts completely by 10 days (Table 2). As shown in Figure 4, both animals achieved successful lymphohematopoietic engraftment, and stable levels of thymic and peripheral chimerism were maintained in these animals even after skin grafting. The significance of these skin graft survival times is discussed later here.
Engraftment in animals receiving high-dose single haplotype-mismatched PBSC infusion

700 cGy TI. The same protocol (700 cGy TI, T-cell depletion, and 100 or $150 \times 10^{8} / \mathrm{kg}$ donor PBSCs) was applied to two single haplotype-mismatched recipients. One of these animals (no. 12885) showed a level of $5-10 \%$ lymphocyte chimerism on day 30 . However, after cessation of the CyA, this level gradually decreased, and, despite an additional infusion of $20 \times$ $10^{8} / \mathrm{kg}$ mobilized PBSCs from the same donor, chimerism could not be detected after day 50 .

a
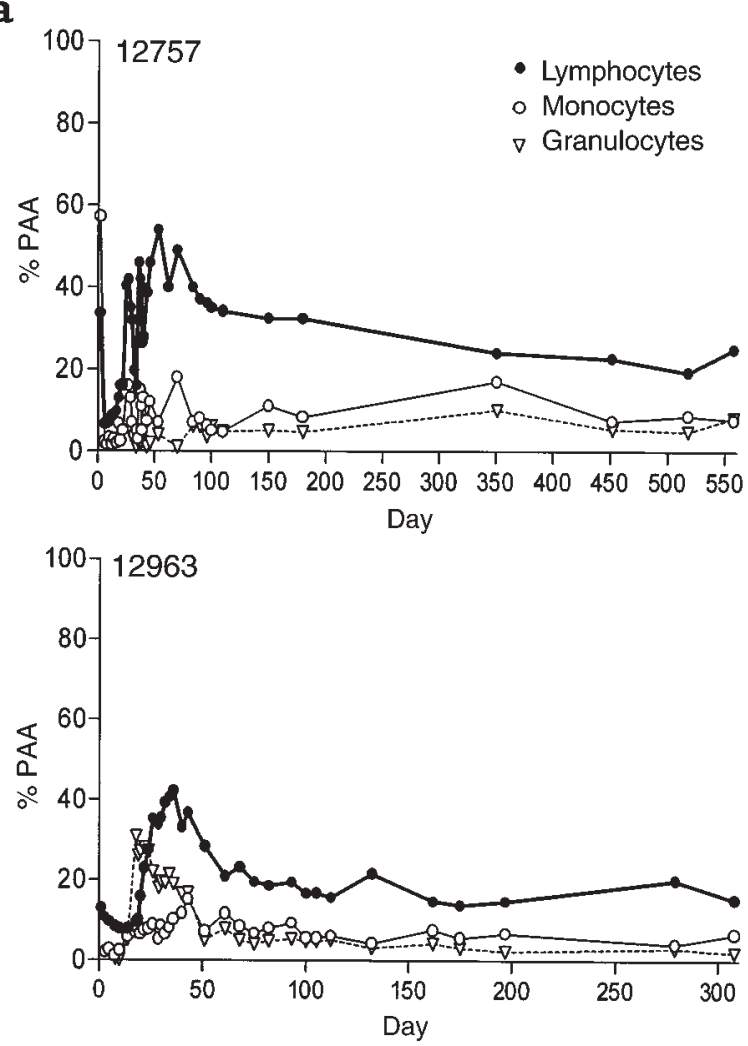

b

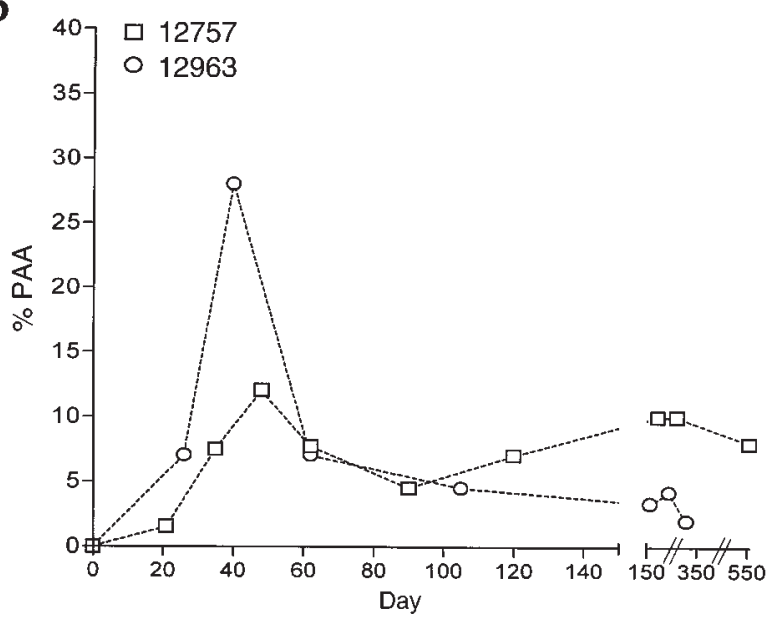

Figure 4

Chimerism among animals receiving SLA-matched PBSCT. (a) Peripheral blood; (b) thymus. 
a

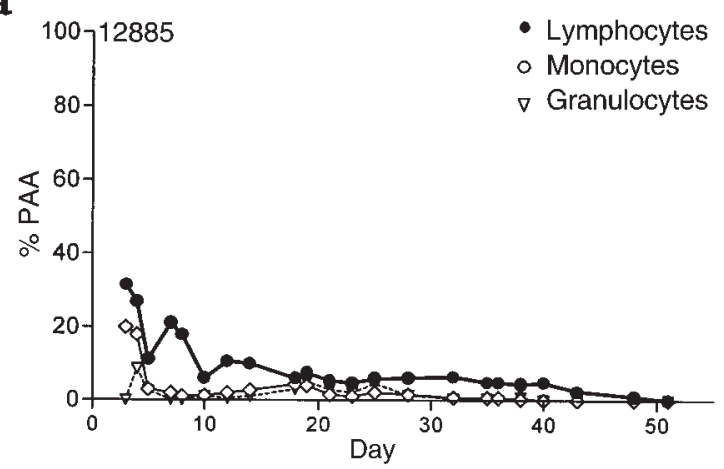

b

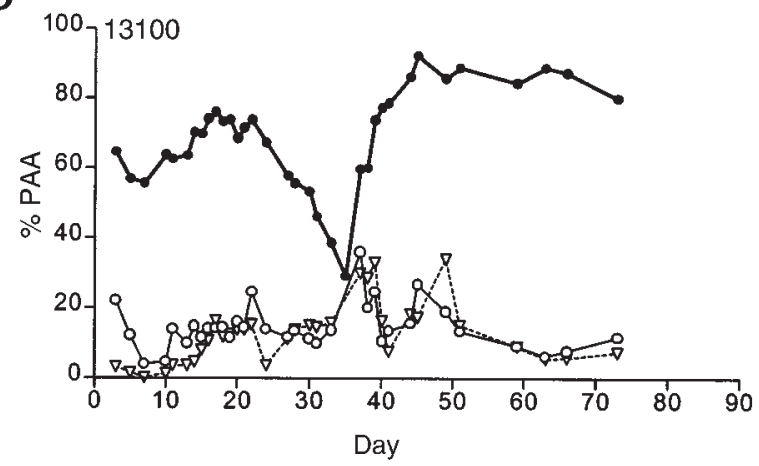

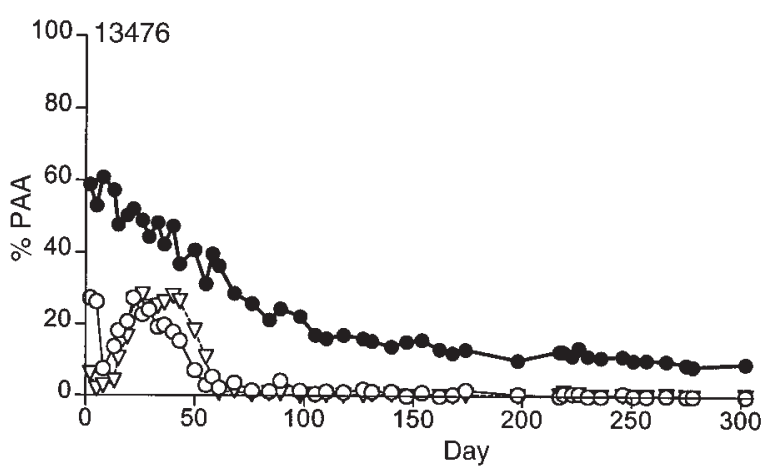

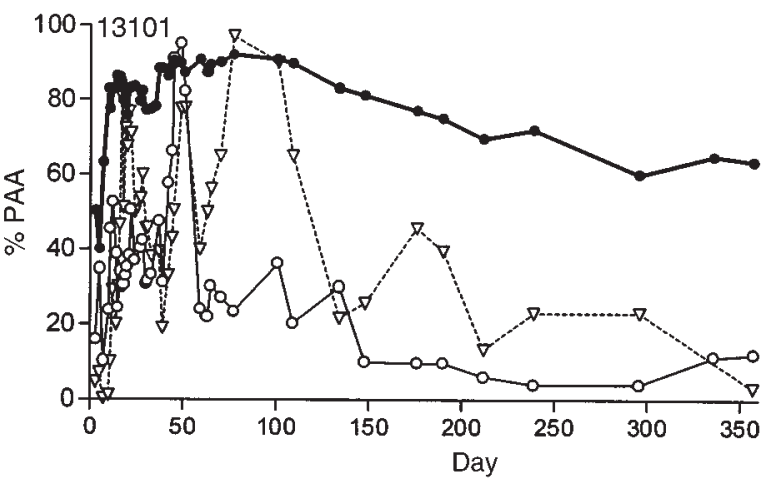

Figure 5

Peripheral chimerism among animals receiving one-haplotype-mismatched PBSCT with (a) 700 cGy TI; (b) 1,000 cGy TI.

Chimerism in the myeloid lineage was barely detectable from the beginning (Figure 5a). In addition, thymic chimerism in this animal was never detectable, even though the thymus appeared healthy and growing on days 38 and 45 as assessed by visualization at biopsy.

In the second animal (no. 13476), engraftment was achieved with a high level of lymphoid chimerism (50-60\%) from the beginning. Myeloid chimerism stabilized to approximately $25-30 \%$ by day 20 , but then fell after day 50 to become almost undetectable. Thymic chimerism was detected in biopsies performed on day $28(15 \%)$, rose by day $40(40 \%)$, but fell again on day 60 (14\%) and was absent by day 98. Chimerism in lymphoid lineages persisted in this animal after cessation of CyA on day 30, but appeared to be gradually decreasing by day 300 (Figure 5a). No clinical signs of GvHD have been observed.

$T I(1,000 c G y)$. The results described here suggested that the preparative regimen was sufficient to permit engraftment of MHC-matched PBSCs, but was probably at the threshold for permitting engraftment across an MHC barrier. We have previously studied lymphohematopoietic engraftment in other species conditioned with a regimen of sublethal WBI plus TI. In both mice (1) and monkeys (34), we found that successful engraftment could be achieved across MHC barriers using $300 \mathrm{cGy}$ WBI and 700 cGy TI. In these regimens, the total irradiation to the thymus is $1,000 \mathrm{cGy}$. We reasoned that the failure of engraftment in animal no. 12885 may have been due to insufficient total TI.
Therefore, the level of TI in this "no WBI" regimen was increased to $1,000 \mathrm{cGy}$ for subsequent single haplotype SLA-mismatched animals.

Two animals (nos. 13100 and 13101) treated with 1,000 cGy TI, T-cell depletion, 30 days of CyA, and 100 $\times 10^{8}$ to $200 \times 10^{8} / \mathrm{kg}$ donor PBSCs both engrafted and developed mixed chimerism (Figure 5b). Animal no. 13100 showed $60-70 \%$ chimerism for the first 3 weeks, but this level decreased dramatically after cessation of CyA on day 30. Shortly thereafter, the animal developed a high fever, a high WBC count, and became less active, presumably reflecting the onset of GvHD (see later here). This animal recovered spontaneously by day 36. Concurrent with recovery, donor lymphocyte chimerism rapidly increased, and 70-80\% lymphocyte chimerism was achieved and maintained from day 40 to the time of sacrifice on day 73. The level of chimerism in the myeloid lineages was lower than in the lymphocyte lineage (10-20\%) at all periods (Figure $5 b)$. Thymic chimerism in this animal reached $4-7 \%$ by day 35 , at which time the animal showed thymic atrophy, probably induced by GvHD.

Animal no. 13101 achieved 80\% donor lymphocyte chimerism by the second week and maintained this level for more than 120 days. Donor chimerism in the myeloid lineage increased by the second week and was subsequently maintained at $40-50 \%$ until day 40 . At this time, the animal showed a transient increase $(80 \%)$ in myeloid chimerism through day 60 , after which granulocyte chimerism increased and mono- 
cyte chimerism decreased (Figure 5b). Thymic chimerism in this animal also reached $4-7 \%$ by day 35 , at which time the animal also showed thymic atrophy. However, all signs of GvHD resolved by day 90, and the thymus began to grow, achieving normal size and $20 \%$ thymic chimerism by day 158 (Figure 6, a-e). Figure 6a shows that donor PAA+ thymocytes determined by FACS were present among the immature CD1-positive thymocytes and among the mature CD3-high thymocytes. The animal remained a mixed chimera for more than 400 days after PBSCT, with $60 \%$ donor lymphocytes, $10 \%$ donor monocytes, and $5 \%$ donor granulocytes.

Histology. Figure 6 (b-e) shows the immunohistochemical staining of a thymic biopsy from animal no. 13101 on day 158 after transplantation. H\&E staining revealed normal thymic structure with well-developed cortex and medulla (Figure 6b). Using a donor-specific class I mAb, donor cells were clearly seen in the medullary region (brown cells in Figure 6c). Double staining with anti-donor class I (blue) and cytokeratin (brown) in Figure 6d demonstrated that the donor cells in the medulla were not epithelial cells, whereas double staining for donor class I (blue) and pan-pig class II (brown) in Figure 6e demonstrated that the donor cells were class II positive, and their morphology was consistent with a dendritic cell type (Figure 6e).

In both of these animals, clinical signs of GvHD, including grade 1-2 skin rash and diarrhea, were observed immediately after the cessation of CyA administration. The skin rash disappeared spontaneously in both animals. However, animal no. 13100 had to be sacrificed on day 73 owing to persistent diarrhea. The pathological examination confirmed that this animal suffered the complications of chronic GvHD, and not of toxicity due to the preparative regimen. In animal no. 13101, the diarrhea disappeared spontaneously, and the animal appeared healthy by day 90 and has remained healthy for more than 400 days.

Induction of tolerance as assessed by renal allografts. In two of these long-term mixed chimerism animals (nos. 13101 and 13476), we tested induction of tolerance by transplantation of an MHC donor-matched renal allograft. Animal nos. 13101 and 13476 received a donor-matched kidney allograft, on post-PBSCT days 190 and 98, respectively. In both cases, the PBSC donor and the kidney allograft donor were different animals, presumably differing for multiple minor antigen disparities. In both cases, the kidneys were accepted long term without the use of immunosuppressive medications after kidney transplantation. As shown in Figure $7 \mathrm{a}$, both animals demonstrated a transient rise in plasma creatinine on postoperative day (POD) 17, as has been reported previously in this model for SLA-matched renal allografts differing for minor antigen disparities (35). Thereafter the creatinines stabilized, and the animals continued to enjoy normal renal function with plasma creatinine levels at less than $1.5 \mathrm{mg} / \mathrm{dL}$ for more than 120 days. In both animals, kidney biopsies demonstrated minimal interstitial infiltration with mononuclear cells on PODs 30,60, and 100. These findings are in contrast to previously reported data for animals receiving onehaplotype-mismatched kidney allografts without immunosuppression, all of which rejected kidney allografts by day 12 (36). A representative control animal (no. 10653) is shown in Figure 7a.

In vitro evidence for tolerance and demonstration of immunocompetence. Figure 7 (b-e) shows the CML results for animal no. 13101(SLA $\left.{ }^{\text {ad }}\right)$, in comparison to the responses of a naive host type animal $\left(\mathrm{SLA}^{\mathrm{ad}}\right)$ run as a control in each assay (Figure $7 \mathrm{~b}$ ). As also seen in Figure 7, animal no. 13101 demonstrated significant anti-donor $S_{L A}{ }^{a c}$ response (> 20\%) before receiving PBSCs (Figure 7c). However, after PBSC administration, both before (Figure $7 \mathrm{~d}$ ) and after (Figure 7e) the kidney allograft, this animal demonstrated donorspecific unresponsiveness in CML. Immunocompetence was demonstrated in all cases by a high anti-third-party response to Yucatan miniature swine (Charles River Breeding Lab, Windham, Maine, USA). The mixed lymphocyte reaction results likewise demonstrated significant anti-donor responses before administration of PBSCs, and donor-specific unresponsiveness and immunocompetence after administration of PBSCs (data not shown).

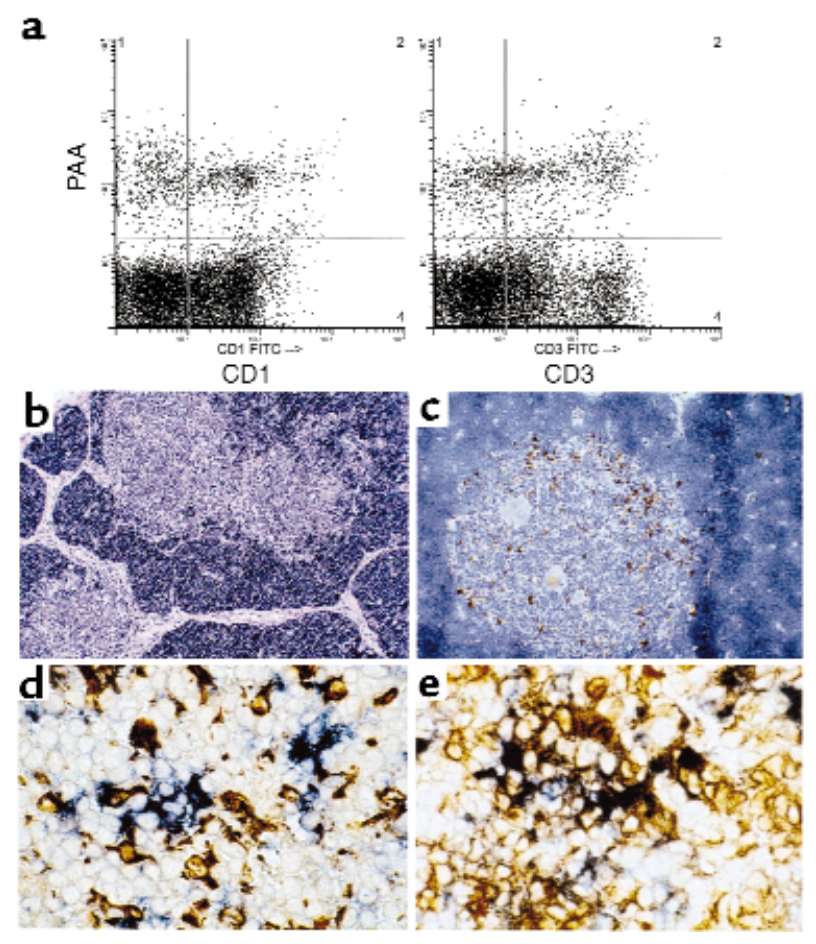

Figure 6

Chimerism detected by flow cytometry and immunohistochemistry in thymic biopsy tissue from animal no. 13101 on day 158 . (a) Double staining of thymus by FACS. (b-e) Histology (immunohistochemistry). 


\section{Discussion}

Protocols for the induction of lymphohematopoietic chimerism through BMT have generally included WBI as part of the preparative regimen $(37,38)$. Even in situations in which immunosuppression of the recipient was achieved by the use of anti-T-cell antibodies, it has generally been thought that some level of WBI was still necessary to make "room" for the donor hematopoietic stem cell graft $(1,37)$. However, it has recently been demonstrated that this requirement for room can be overcome by increasing the number of donor hematopoietic stem cells administered, the so-called "megadose" approach $(8,9)$. This approach was demonstrated first for syngeneic bone marrow grafts $(8,9)$ and was subsequently shown to permit fully mismatched allogeneic marrow engraftment if conditioned with $\mathrm{TI}$ and recipient $\mathrm{T}$-cell depletion (11). The data are consistent with the possibility that niches for the engraftment of administered stem cells are filled by mass action, and that the effect of low-dose irradiation is to empty some of the niches, shifting the equilibrium in favor of adminis- tered cells. On the other hand, such irradiation would not be required if the donor inoculum were sufficiently large. An alternative explanation for these results might be that WBI serves to provide sufficient host immunosuppression to allow donor cells to engraft by creating their own space (2). However, the previous demonstration of a comparable effect of megadoses for syngeneic bone marrow $(8,9)$ would be more consistent with the mass action hypothesis.

Despite the excitement generated by the possibility of avoiding myeloablation in BMT protocols, the applicability of the megadose approach to large animals and human beings was considered limited, as the doses of bone marrow used were 10-100 times the usual dose, requiring harvests that would clearly not be feasible from living donors. However, the advent of PBSC technology has made it possible to obtain such megadose quantities of stem cells from living donors by stimulation with hematopoietic cytokines followed by leukapheresis (39). Thus, in the present studies, leukapheresis on 3 consecutive days, after 1 week of cytokine administration, allowed us

\section{Figure 7}

(a) Plasma creatinine levels of single haplotype-mismatched chimeric re cipients that received donor MHCmatched kidney allografts without immunosuppression: Animals no. 13101 and no. 13476 were chimeric animals that received donor $\mathrm{MHC}$ matched kidney allografts; animal no. 10653 was a naive animal that received a single haplotype-mismatched kidney allograft. (b-e) Donor-specific tolerance and immunocompetence of single haplotype-mismatched chimeric animal no. 13101: CTL responses of a naive SLA ad animal (b) and of animal no. 13101 before PBSCT (c); after PBSCT, but before kidney transplantation (KTx) (d); and after KTx (e). Each graph shows anti-self SLA ad (open squares), anti-donor SLA ${ }^{\text {ac }}$ (closed squares), and anti-third-party Yucatan miniature swine (open triangles) response.
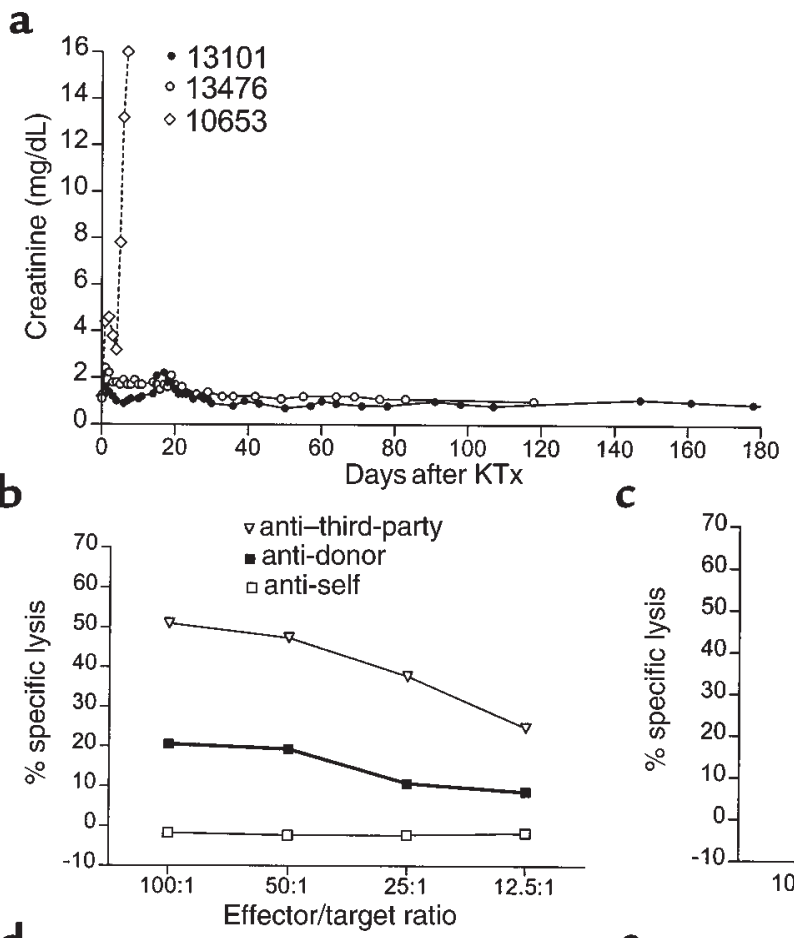

C
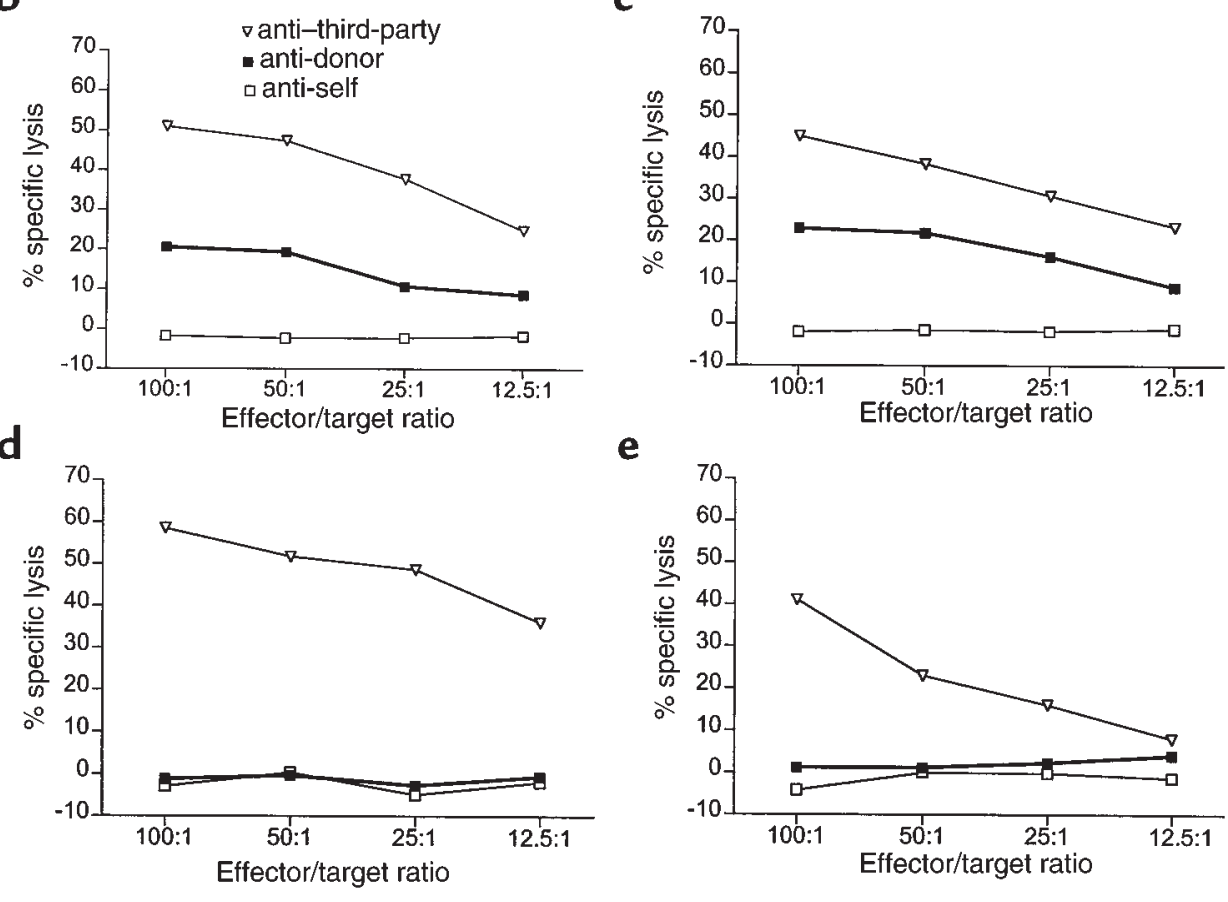
to achieve a collection of $1.8 \times 10^{11}$ mononuclear cells, more than 50 times the number usually harvested from the bone marrow of comparable living donor animals. As demonstrated by the histology in Figure $6(\mathrm{~b}-\mathrm{e})$, tolerance induced by these cells most likely involves central deletion by donor dendritic cells in the thymus. As demonstrated here, tolerance has been confirmed in these animals by acceptance of either skin grafts or vascularized organ allografts without immunosuppression.

Another important facet of the preparative regimen required to achieve engraftment without WBI, is effective depletion of mature $\mathrm{T}$ cells from the recipient. Although such depletion has been possible with antisera and with mAb's in mice (40), similar depletion in large animals and humans has been difficult to achieve $(41,42)$. Previous studies in miniature swine have shown coating of peripheral $\mathrm{T}$ cells by such antibodies, without effective depletion (42). However, the use of a toxin-conjugated anti-CD3 mAb (pCD3-CRM9) has now enabled depletion to an extent compatible with engraftment (22). Use of a similar immunotoxin has been demonstrated to be effective in nonhuman primates (43-45). Clinical protocols may eventually rely on such immunotoxins, or may be possible with new mAb's that have shown markedly effective T-cell depletion in humans $(46,47)$ but which unfortunately cannot be tested in nonhuman primates, as they are species-specific. Although CD3 immunotoxins have been reported to cause vascular leak syndrome in some protocols $(32,33)$, no vascular leak syndrome has been observed in miniature swine treated with PCD3-CRM9 in this nonmyelosuppressive regimen without WBI.

Depletion of mature recipient and donor $\mathrm{T}$ cells is achieved in mice by the use of high doses of unconjugated mAb's. These antibodies remain active in the recipient plasma for 2 weeks after administration (48) and are thought to be responsible for subsequent depletion of donor T cells in the bone marrow inoculum, thus preventing GvHD. In contrast, T-cell depletion with PCD3-CRM9 involves treatment with a low dose of conjugated antibody, which is no longer active in the plasma by the time that donor PBSCs are administered (data not shown). We have therefore utilized a 30-day course of CyA in the present protocol in an attempt to prevent the induction of GvHD by donor $\mathrm{T}$ cells. This treatment was effective in preventing GvHD in recipients of SLA-matched transplants, but was only partially effective for SLA-mismatched transplants. We have therefore subsequently increased CyA treatment by tapering over an additional 30 days in the case of mismatched transplantation, and our preliminary data indicate that this additional treatment is effective in preventing this complication (Y. Fuchimoto et al., manuscript in preparation). In addition to its role in preventing GvHD, CyA may also help to facilitate engraftment by preventing residual mature $T$ cells among host thymocytes from causing intrathymic rejection of donor cells (see later here).
Our data are consistent with previous murine studies and recent dog studies indicating that bone marrow space is not an absolute requirement for lymphohematopoietic engraftment $(2,8,9,11)$. However, the importance of TI as part of the preparative regimen suggests that thymic space or elimination of thymic alloreactivity is an absolute requirement for the achievement of engraftment of MHC-mismatched marrow, a finding also supported by previous data in mice $(1,9,48,49)$. Thus, in one of the two animals treated with only 700 cGy of TI, initial engraftment was lost shortly after treatment with CyA that was discontinued on day 30, and in the other animal, engraftment was more persistent but nevertheless not as stable as in animals treated with $1,000 \mathrm{cGy}$ of TI. We reasoned that for MHC-mismatched reconstitution, additional TI might be necessary, as it is known that antibody-mediated depletion is less effective in the thymus than peripherally $(1,22)$. When TI was increased to $1,000 \mathrm{cGy}$, the same total thymic dose received in nonmyeloablative protocols incorporating 300 cGy WBI plus 700 cGy TI was achieved (1). In this setting, both recipients of one haplotype MHC-mismatched marrow engrafted. Immunological sites in addition to the thymus may be affected by the TI as administered. These sites would undoubtedly include some marrow tissue in the nearby ribs, vertebrae, and sternum, as well as the blood recirculating through the radiotherapy field during the course of irradiation. This additional irradiation could potentially affect engraftment by creating some marrow space or by suppressing the immune system to some degree. However these effects, if present, are clearly much less myelosuppressive and immunosuppressive than those of WBI. Thus, unlike recipients of sublethal WBI (17), none of the animals receiving TI without WBI required any blood support or developed any signs of immunodeficiency after transplantation. Recent data in mice show that costimulatory blockade with CTLA4Ig and/or antiCD40L antibodies is capable of enabling lasting engraftment without TI, probably because costimulatory blockade obviates the need for complete T-cell depletion (50). Therefore, we may be able to eliminate all irradiation from the preparative regimen by incorporating costimulatory blockade into our no-WBI regimen, and studies along these lines are currently in progress.

One animal in this study demonstrated prolonged donor skin graft survival after an MHC-matched PBSC infusion, with eventual rejection of the skin graft by day 45. However, this animal nevertheless maintained peripheral and thymic mixed chimerism and accepted a heart transplant from the same donor (51). These data suggest that despite the induction of central tolerance by donor PBSCs in this animal, skin-specific antigens to which the recipient was not tolerized by PBSCs were present on the donor skin graft. The other animal tested in the same way showed permanent donor skin survival. These data are consistent with what is known about skin-specific antigens in mice (52-54). Because it is likely that only a few alleles for such skin-specific anti- 
gens exist within our swine herd, some animals could be expected to share common alleles by chance, and therefore a certain percentage of chimeric animals might be expected to accept skin grafts, whereas others would reject. The subject of skin-specific antigens in miniature swine will be discussed in detail elsewhere (Y. Fuchimoto, manuscript in preparation).

The ability to achieve hematopoietic stem cell engraftment without WBI has many advantages for clinical applications. Previous studies in miniature swine, using WBI as part of the preparative regimen, have produced pancytopenia over the first 2 weeks after bone marrow administration, necessitating blood product support (55). Similar blood product support is required in most large animal models of BMT, as well as in humans $(31,56,57)$. However, as seen in the present studies, when engraftment and mixed chimerism are obtained without WBI, the recipient animals do not develop sufficient pancytopenia to require blood product support. Although the dangers of pancytopenia are acceptable in the treatment of hematologic malignancies, they pose a major impediment to the use of BMT in the treatment of less immediately life-threatening diseases, such as hemoglobinopathies. Likewise, if tolerance induction by BMT were possible without significant myelosuppression, our data indicate that this protocol might be applicable to the treatment of endstage organ failure by organ transplantation. We therefore believe that the extension of the "no WBI" approach to the establishment of mixed chimerism across MHC barriers may have important implications for the treatment of these non-life-threatening diseases through hematopoietic reconstitution.

\section{Acknowledgments}

The authors thank T. Kawai and B. Nikolic for their critical review of the manuscript; A. Griesemer, A. Hansen, and K. Allison for expert animal care; J. Sachs for editorial assistance; and L.A. Bernardo for help in manuscript preparation. The authors also acknowledge McGaw, Inc. for generously providing FreAmine III 10\%, Lloyd Pharmaceuticals for providing Xylazine, Abbott Laboratories for providing Ancef and Liposyn III 20\%, OrthoMcNeil Pharmaceuticals for providing Ofloxin, and Novartis Pharmaceuticals for providing Neoral Oral Soln. This work was supported in part by National Institutes of Health (grants 5RO1 AI-39755 and 2PO1 HL-18646) and by the Yates Charitable Foundation.

1. Sharabi, Y., and Sachs, D.H. 1989. Mixed chimerism and permanent specific transplantation tolerance induced by a nonlethal preparative regimen. J. Exp. Med. 169:493-502.

2. Storb, R., et al. 1999. Stable mixed hematopoietic chimerism in dog leukocyte antigen-identical littermate dogs given lymph node irradiation before and pharmacologic immunosuppression after marrow transplantation. Blood. 94:1131-1136.

3. Walters, M.C., et al. 1996. Bone marrow transplantation for sickle cell disease. N. Engl. J. Med. 335:369-376.

4. Walters, M.C., et al. 1996. Barriers to bone marrow transplantation for sickle cell anemia. Biol. Blood Marrow Transplant. 2:100-104.

5. Storb, R., et al. 1998. Current and future preparative regimens for bone marrow transplantation in thalassemia. Ann. NY Acad. Sci. 850:276-287.
6. Ildstad, S.T., and Sachs, D.H. 1984. Reconstitution with syngeneic plus allogeneic or xenogeneic bone marrow leads to specific acceptance of allografts or xenografts. Nature. 307:168-170.

7. Sykes, M., and Sachs, D.H. 1988. Mixed allogeneic chimerism as an approach to transplantation tolerance. Immunol. Today. 9:23-27.

8. Stewart, F.M., Crittenden, R.B., Lowry, P., Pearson-White, S., and Quesenberry, P.J. 1993. Long-term engraftment of normal and post-5-fluorouracil murine marrow into normal nonmyeloablated mice. Blood. 81:2566-2571.

9. Sykes, M., Szot, G.L., Swenson, K., Pearson, D.A., and Wekerle, T. 1998. Separate regulation of peripheral hematopoietic and thymic engraftment. Exp. Hematol. 26:457-465.

10. Bachar-Lustig, E., Rachamim, N., Li, H.-W., Lan, F., and Reisner, Y. 1995. Megadose of T cell-depleted bone marrow overcomes MHC barriers in sublethally irradiated mice. Nat. Med. 1:1268-1273.

11. Sykes, M., Szot, G.L., Swenson, K.A., and Pearson, D.A. 1997. Induction of high levels of allogeneic hematopoietic reconstitution and donor-specific tolerance without myelosuppressive conditioning. Nat. Med. 3:783-787.

12. Soderling, C.C.B., Song, C.W., Blazar, B.R., and Vallera, D.A. 1985. A correlation between conditioning and engraftment in recipients of MHCmismatched $\mathrm{T}$ cell-depleted murine bone marrow transplants. J. Immunol. 135:941-946.

13. Gutstein, N.L., and Wofsy, D. 1986. Administration of F(ab')2 fragments of monoclonal antibody to L3T4 inhibits humoral immunity in mice without depleting L3T4+ cells. J. Immunol. 137:3414-3419.

14. Vallera, D.A., Soderling, C., Carson, G., and Kersey, J. 1981. Bone marrow transplantation across major histocompatibility barriers in mice: the effect of elimination of $\mathrm{T}$ cells from donor grafts by treatment with monoclonal Thy1.2 plus complement or antibody alone. Transplantation. 31:218-222.

15. Kernan, N.A., Flomenberg, N., Dupont, B., and O'Reilly, R.J. 1987. Graft rejection in recipients of T-cell-depleted HLA-nonidentical marrow transplants for leukemia. Transplantation. 43:842-847.

16. Storb, R., et al. 1997. Stable mixed hematopoietic chimerism in DLAidentical littermate dogs given sublethal total body irradiation before and pharmacological immunosuppression after marrow transplantation. Blood. 89:3048-3054.

17. Huang, C.A., et al. 2000. Stable mixed chimerism and tolerance using a non-myeloablative preparative regimen in a large animal model. J. Clin. Invest. 105:173-181.

18. Sachs, D.H., et al. 1976. Transplantation in miniature swine. I. Fixation of the major histocompatibility complex. Transplantation. 22:559-567.

19. Pennington, L.R., Lunney, J.K., and Sachs, D.H. 1981. Transplantation in miniature swine. VIII. Recombination within the major histocompatibility complex of miniature swine. Transplantation. 31:66-71.

20. Colby, C., et al. 2000. Cytokine mobilized peripheral blood progenitor cells for allogeneic reconstitution of miniature swine. Transplantation. 69:135-140.

21. Kozlowski, T., et al. 1999. Effect of pig-specific cytokines on mobilization of hematopoietic progenitor cells in pigs and on pig bone marrow engraftment in baboons. Xenotransplantation. 6:17-27.

22. Huang, C.A., et al. 1999. In vivo T cell depletion in miniature swine using the swine CD3 immunotoxin, pCD3-CRM9. Transplantation. 68:855-860.

23. Pescovitz, M.D., Lunney, J.K., and Sachs, D.H. 1984. Preparation and characterization of monoclonal antibodies reactive with porcine PBL. J. Immunol. 133:368-375.

24. Huang, C.A., et al. 1999. Characterization of a monoclonal anti-porcine CD3 antibody. Xenotransplantation. 5:201-212.

25. Saalmuller, A., et al. 1996. The second international swine CD workshop. Vet. Immunol. Immunopathol. 54:155-158.

26. Fuchimoto, Y., et al. 1999. An allelic non-histocompatibility antigen with wide tissue distribution as a marker for chimerism in pigs. Tissue Antigens. 54:43-52.

27. Ivansoka, D., Sun, D.C., and Lunney, J.K. 1991. Production of monoclonal antibodies reactive with polymorphic and monomorphic determinants of SLA class I gene products. Immunogenetics. 33:220-223.

28. Leight, G.S., et al. 1978. Transplantation in miniature swine. III. Effects of MSLA and A-O blood group matching on skin allograft survival. Tissue Antigens. 12:65-74.

29. Kirkman, R.L., Colvin, R.B., Flye, M.W., Williams, G.M., and Sachs, D.H. 1979. Transplantation in miniature swine. VII. Evidence for cellular immune mechanisms in hyperacute rejection of renal alografts. Transplantation. 28:24-30.

30. Kortz, E.O., et al. 1990. Mechanism of tolerance following class I disparate renal allografts in miniature swine: cellular responses of tolerant animals. Transplantation. 49:1142-1149.

31. Pennington, L.R., et al. 1988. Bone marrow transplantation in miniature swine. I. Development of the model. Transplantation. 45:21-26.

32. Thrush, G.R., Lark, L.R., Clinchy, B.C., and Vitetta, E.S. 1996. Immunotoxins: an update. Annu. Rev. Immunol. 14:49-71. 
33. Contreras, J.L., et al. 1998. Peritransplant tolerance induction with antiCD3-immunotoxin: a matter of proinflammatory cytokine control. Transplantation. 65:1159-1169.

34. Kawai, T., et al. 1995. Mixed allogeneic chimerism and renal allograft tolerance in cynomolgus monkeys. Transplantation. 59:256-262

35. Rosengard, B.R., Ojikutu, C.A., Fishbein, J., Kortz, E.O., and Sachs, D.H 1992. Selective breeding of miniature swine leads to an increased rate of acceptance of MHC-identical, but not of class-I disparate, renal allografts. J. Immunol. 149:1099-1103.

36. Gianello, P.R., et al. 1995. Induction of tolerance to renal allografts across single-haplotype MHC disparities in miniature swine. Transplan tation. 59:884-890.

37. Tomita, Y., Sachs, D.H., and Sykes, M. 1994. Myelosuppressive conditioning is required to achieve engraftment of pluripotent stem cells contained in moderate doses of syngeneic bone marrow. Blood. 83:939-948.

38. Popitz-Bergez, F.A., et al. 1988. Bone marrow transplantation in miniature swine. II. Effect of selective genetic differences on marrow engraftment and recipient survival. Transplantation. 45:27-31.

39. Aversa, F., et al. 1994. Successful engraftment of T-cell-depleted haploidentical "three-loci" incompatible transplants in leukemia patients by addition of recombinant human granulocyte colony-stimulating factor-mobilized peripheral blood progenitor cells to bone marrow inoculum. Blood. 84:3948-3955.

40. Cobbold, S.P., Martin, G., Qin, S., and Waldmann, H. 1986. Monoclonal antibodies to promote marrow engraftment and tissue graft tolerance. Nature. 323:164-166.

41. Kawai, T., Wong, J., MacLean, J., Cosimi, A.B., and Wee, S.L. 1994. Characterization of a monoclonal antibody (6G12) recognizing the cynomolgus monkey CD3 antigen. Transplant. Proc. 26:1845-1846.

42. Suzuki, T., Sundt, T.M., Mixon, A., and Sachs, D.H. 1990. In vivo treatment with anti-porcine T-cell antibodies. Transplantation. 50:76-81.

43. Neville, D.M.J., et al. 1996. A new reagent for the induction of T-cell depletion, anti-CD3-CRM9. J. Immunother. Emphasis Tumor Immunol. 19:85-92.

44. Thomas, J.M., et al. 1997. Preclinical studies of allograft tolerance in rhesus monkeys: a novel anti-CD3-immunotoxin given peritransplant with donor bone marrow induces operational tolerance to kidney allografts. Transplantation. 64:124-135
45. Knechtle, S.J., et al. 1998. Primate renal transplants using immunotoxin. Surgery. 124:438-446.

46. Przepiorka, D., et al. 1998. A phase II study of BTI-322, a monoclonal anti-CD2 antibody, for treatment of steroid-resistant acute graft-versushost disease. Blood. 92:4066-4071.

47. Dumont, C., et al. 1998. Potent apoptotic signaling and subsequent unresponsiveness induced by a single CD2 mAb (BTI-322) in activated human peripheral T cells. J. Immunol. 160:3797-3804.

48. Tomita, Y, Khan, A., and Sykes, M. 1996. Mechanism by which additional monoclonal antibody $(\mathrm{mAB})$ injections overcome the requirement for thymic irradiation to achieve mixed chimerism in mice receiving bone marrow transplantation after conditioning with anti-T cell $\mathrm{mABs}$ and 3-Gy whole body irradiation. Transplantation. 61:477-485.

49. Tomita, Y., Sachs, D.H., Khan, A., and Sykes, M. 1996. Additional monoclonal antibody $(\mathrm{mAB})$ injections can replace thymic irradiation to allow induction of mixed chimerism and tolerance in mice receiving bone marrow transplantation after conditioning with anti-T cell mABs and 3-Gy whole body irradiation. Transplantation. 61:469-477.

50. Wekerle, T., and Sykes, M. 1999. Mixed chimerism as an approach for the induction of transplantation tolerance. Transplantation. 68:459-467.

51. Schwarze, M.L., et al. 2000. Mixed hematopoietic chimerism induces long term tolerance to cardiac allografts in miniature swine. Ann. Thorac. Surg. In press.

52. Boyse, E.A., Lance, E.M., Carswell, E.A., Cooper, S., and Old, L.J. 1970. Rejection of skin allografts by radiation chimaeras: selective gene action in the specification of cell surface structure. Nature. 227:901-903.

53. Boyse, E.A., Carswell, E.A., Scheid, M.P., and Old, L.J. 1973. Tolerance of Sk-incompatible skin grafts. Nature. 244:441-442.

54. Steinmuller, D., and Lofgreen, J.S. 1974. Differential survival of skin and heart allografts in radiation chimaeras provides further evidence for Sk histocompatibility antigen. Nature. 248:796-797.

55. Sachs, D.H. 1992. MHC homozygous miniature swine. In Swine as models in biomedical research. M.M. Swindle, D.C. Moody, and L.D. Phillips, editors. Iowa State University Press. Ames, Iowa, USA. 3-15.

56. Ladiges, W.C., Storb, R., and Thomas, E.D. 1990. Canine models of bone marrow transplantation. Lab. Anim. Sci. 40:11-15.

57. Atkins, K. 1990. Reconstruction of the haemopoietic and immune systems after marrow transplantation. Bone Marrow Transplant. 5:209-226. 